\title{
LOS NUEVOS ESCENARIOS RURALES: DE LA AGRICULTURA A LA MUTIFUNCIONALIDAD ${ }^{1}$
}

\author{
Encarnación Aguilar CRiado \\ Universidad de Sevilla ${ }^{2}$
}

Resumen: Este texto analiza los principales elementos de transformación de la sociedad rural europea como consecuencia de los efectos de la modernización agrícola de mediados del siglo XX. Los principios que actualmente caracterizan a la nueva ruralidad fueron construidos a partir de ellos. El argumento central de este texto es que los cambios operados en la sociedad rural deben relacionarse con el contexto macroeconómico y social en el que está inmersa, rompiendo por tanto con la clásica dicotomía ruralurbano. De la misma forma, la transformación de esta realidad social aparece vinculada con los paradigmas científicos desde los que se ha estudiado. La evolución de la sociedad rural ha tenido una fuerte incidencia en la trayectoria seguida por los Estudios Rurales, cuyos principales fundamentos son igualmente analizados.

PALABRAS ClAVE: nueva ruralidad, estudios rurales, desarrollo rural, antropología, sociedades rurales.

AвSTRACT: This paper analyzes the main elements of transformation of European rural society as a result of the effects of agricultural modernization in the mid twentieth century. The principles that currently characterize the new rurality were built from. The central argument of this paper is to link the changes in rural society with macroeconomic and social context in which it is embedded, thus breaking with the traditional

${ }^{1}$ Este trabajo se inscribe en el grupo de investigación TECUDE «Territorio, Cultura y Desarrollo». Forma parte de los resultados del proyecto «La producción de calidad: nuevas estrategias rurales para nuevos consumidores». I+D. Ministerio de Economía y Competitividad y Fondos Feder (CSO2010-22074-C03-01). Su contenido es el fruto de ańos dedicados a los estudios rurales. No hubiera sido posible sin la inestimable ayuda de mi equipo de investigación. A Carmen Lozano y Alberto Pérez, por su minuciosa lectura del primer borrador. A Ignacio López, Santiago Amaya y Paula Felizón mi agradecimiento por todas sus aportaciones, su apoyo y su amistad. A los alumnos del Grado de Antropología Social y Cultural de la Universidad de Sevilla de la asignatura de Antropología y Sociedades Rurales, que me han obligado a sistematizar datos y lecturas, mi agradecimiento por su entusiasmo y su compromiso con la asignatura.

2 eaguilar@us.es 
rural-urban dichotomy. In this sense the changes in social reality are also linked with scientific paradigms from which rurality were analyzed. The transformation of rural society has led to the development of Rural Studies, whose main bases are also discussed.

KEYwORDs: new ruralities, rural studies, rural development, anthropology, rural society.

\section{Los campesinos y los estudios rurales}

Los estudios rurales conforman en la actualidad un campo científico de larga trayectoria multidisciplinar. Esta característica pasa por ser uno de los distintivos de esta subdisciplina, cuyos principales paradigmas son el resultado de las contribuciones efectuadas por distintos campos de las ciencias sociales: antropología, sociología, economía, geografía e historia. La aparición de la revista Journal of Peasant Studies en 1973 es el mejor reflejo de la riqueza conceptual de un campo de estudio, que inició su andadura precisamente para explicar la crisis de una sociedad rural que desaparecía a fines del siglo XIX, al calor de la Revolución Industrial.

Los campesinos constituyen unos de los temas fundacionales de los estudios rurales. El fin del campesinado, tantas veces anunciado, se erigió desde finales del siglo XIX en el lugar común de reflexión de teóricos, tecnócratas y políticos que propugnaban su irremediable desaparición como grupo social, una vez desmantelados los cimientos de la sociedad feudal europea en la que se había desarrollado. Tal y como hemos señalado en otro texto (Aguilar, 1999), el surgimiento de los campesinos como tema de estudio es paralelo al proceso de la industrialización. La gran transformación marcaría un antes y un después en la pretendida permanencia de un mundo rural que se descomponía ante el rápido crecimiento de las ciudades y la emigración, fenómenos que más allá de sus repercusiones sociales a finales del XIX, se instituyeron como nuevos objetos de reflexión intelectual.

Esta preocupación acerca del devenir de un grupo en franca decadencia trataba de diseñar una nueva sociedad que ofreciera alternativas a una población rural, todavía mayoritaria en zonas de capitalismo periférico. En este contexto sociológico, el modelo teórico de Marx proporcionó buena parte de las herramientas analíticas con las que abordar el estudio de los campesinos desde la economía política. Los ejes centrales de este debate se centraron, desde sus inicios, en la ya recurrente idea de desaparición del campesino. Tachar a este tipo 
de población como «supervivencia del pasado» o como "barbarie dentro de la civilización" (Marx, 1867), fueron sólo algunos de los ejemplos del marcado espíritu anticampesinista de Marx que fueron validados por sus más fervientes seguidores. Estudiosos como Kautsky (1899) o políticos como Lenin (1889), que partieron del axioma de que una agricultura familiar no tendría cabida dentro del marco del capitalismo, en la medida en que la concentración de tierras en manos de la burguesía agraria determinaría la expropiación de los campesinos y su proletarización ${ }^{3}$.

En el otro extremo se sitúa la figura de Chayanov. Le debemos a este economista ruso, fundador de la Vieja Tradición de Estudios Campesinos, las páginas más acertadas sobre la especificidad de este tipo de economía. Su racionalidad quedó magistralmente recogida en la ya clásica obra: La Organización de la Unidad Doméstica Campesina (1925). En sus páginas encontramos uno de los análisis más certeros de las bases sobre las que se construye la lógica económica campesina. Una racionalidad que estaba determinada, en esencia, por su doble naturaleza de unidad económica y unidad familiar. En este principio elemental, tal y como el mismo Chayanov señalaba, se sustenta toda su teoría. Este doble carácter de empresa y de familia, que él denominó Unidad de Explotación Doméstica, expresaba el carácter específico de la lógica económica campesina (Chayanov, 1925). En esta ecuación que combina recursos, medios de producción y composición del grupo doméstico se hallan los pilares de la organización económica de la unidad de explotación campesina.

La aportación de Chayanov, largamente silenciada tras su muerte, fue la base sobre la que sustentó, a partir de los años 60 , la orientación de los futuros estudios campesinos y, por extensión, de la economía doméstica. Su enfoque desveló nuevos caminos a la antropología sobre todo a aquellos centrados en el análisis de las economías de subsistencia (Sahlins, 1972) y sentó las bases de los estudios campesinos realizados desde posiciones materialistas, consagrando las obras clásicas de antropólogos (Wolf, 1971; Mintz, 1973; Palerm, 1976), sociólogos (Galesky, 1977; Shanin, 1983) e historiadores (Hobsbawn y Alavi, 1976).

3 Con todo, Marx esbozó las líneas maestras que posteriormente desarrollaron los estudiosos del campesinado, al establecer que la especificidad de la economía campesina era la ausencia del concepto de beneficio, lo que determinaba la incapacidad de acumulación de capital. Esta ausencia del valor del trabajo como mercancía, junto con la teoría de la renta de la tierra, constituían para Marx los dos elementos fundamentales que distinguían al sistema económico campesino del capitalista. 
Le debemos a la riqueza intelectual de este grupo, conocido como La Nueva Escuela de Estudios Campesinos, el desarrollo y la aplicación de las premisas chayanovianas. Las investigaciones realizadas sobre tipologías de campesinos a nivel mundial, y el carácter multidisciplinar de este grupo, no sólo pusieron las bases de los actuales estudios rurales, sino que determinaron algunas de las peculiaridades de este campo científico.

Este grupo supuso además la definitiva ruptura con el paradigma de la cultura folk, dominante en la sociología y la antropología norteamericana desde los años 50. Unos planteamientos que surgieron de una reformulación de los modelos de relaciones duales decimonónicos, para explicar la evolución social desde unos estadios de menor a mayor complejidad. Tales tipos de relaciones tendieron a identificarse con estructuras sociales presentes en dos patrones de asentamiento: el rural y el urbano. El modelo de The Folk-Society (Redfield, 1947), instauraría una concepción de lo rural como un tipo ideal de pequeña comunidad, basada en la tradición oral, dotada de una homogeneidad social y un sentido de solidaridad grupal que delimitaban una cultura característica, cuyos elementos irán debilitándose, hasta su desaparición, con el progresivo tránsito hacia la urbanización, proceso que esquematizaría en su tipología Folk-Urban Continunm.

Precisamente fue al calor de esta orientación como los campesinos pasaron a ser objeto de estudio de los antropólogos, protagonistas centrales de los estudios de comunidad que dominaron la práctica etnográfica de los antropólogos hasta los años 70. Unas investigaciones que, como veremos más adelante, fueron fundamentales para explicar el inicio de nuestra disciplina en España. Este paradigma folk estaba directamente relacionado con la vinculación entre ruralidad y culturas tradicionales, una simbiosis que ha terminado por lastrar este campo científico hasta la actualidad, al ser percibido por nuestros colegas como un espacio de menor rango, y como una disciplina tan rancia como residual es la realidad que estudia (González y Moyano, 2011).

\section{Campesinos, Folk y Estudios de Comunidad}

La naciente antropología española de los años 70 convirtió en objeto prioritario de sus estudios a los campesinos. Por ende, nuestro país pasó a ser una nueva área de estudio para los antropólogos de la corriente folk anglosajona. En este momento, y como resultado de sus primeros trabajos sobre América Latina, George Foster realizó su famoso viaje por España acompañado por Julio Caro 
Baroja entre 1949-1950 (Foster, 1951). La realidad que se encontró fue la de un país empobrecido económica y culturalmente, con una población mayoritariamente rural y una agricultura tradicional. Estas características lo convertían en un lugar idóneo para aplicar la propuesta de la corriente folk norteamericana centrada en el análisis de prácticas económicas, valores, costumbres y comportamientos de las culturas campesinas en una recién descubierta área de estudio para los antropólogos anglosajones: el Mediterráneo. Fue éste el mismo período y contexto académico en el que el inglés Pitt Rivers se instaló en la Sierra de Cádiz, escribiendo la primera monografía antropológica sobre España: The People of the Sierra (1954).

Se trataba, en esencia, de estudios de comunidad que ofrecían generalizaciones culturales extraídas de casos muy concretos. Una realidad apostada en la tradicionalidad de sus instituciones, cuya incipiente crisis obedecía siempre a cambios externos, personalizados en la emigración y urbanismo. De cualquier forma, en este periodo — que en otro lugar hemos denominado Antropología en España sin Antropólogos Españoles (Aguilar, Feixa y Melis, 2000) — merece ser destacado porque en él se concentran numerosas aportaciones que la antropología española ha hecho al campo de los estudios rurales, centrándose sobre todo los campesinos, como grupos tradicionales (Brandes, 1991; Prat, 1992).

La posterior aportación de la economía política de la mano de Wolf y su libro Peasants (1971), fue decisiva para entender la emergencia de una segunda fase de estudios sobre campesinos entre los antropólogos españoles. En esta etapa fue fundamental la influencia que Ángel Palerm ejerció en aquellos jóvenes investigadores, a través de la figura de su hijo Juan Vicente Palerm, así como las intensas relaciones de estos estudiosos con sociólogos y economistas rurales, muy bien representado por la figura y obra de E. Sevilla Guzmán (1979).

La riqueza y el potencial de este grupo contó con una voz propia y de primer nivel, la revista Agricultura y Sociedad. Creada por el Ministerio de Agricultura en 1976, fue el mejor reflejo de la vitalidad de los estudios rurales dentro y fuera de España. La clausura de la publicación en 1998 marcó precisamente el final de esta etapa de estudios rurales ${ }^{4}$. Quizás el último testigo de esta época fueron las dos obras colectivas que bajo el titulo genérico de Agricultura y Sociedad en la España contemporánea (1997) y Agricultura y Sociedad en el cambio de siglo

${ }^{4}$ En 1998 aparecería Revista Española de Estudios Agrosociales y Pesqueros, fundada como refundición de las Revistas: Economía Agraria y Agricultura y Sociedad. 
(2002), siguen siendo referencias importantes en este campo de estudio, porque hallaremos un representativo grupo de autores, temáticas y disciplinas que conformaron los estudios rurales en España durante esas décadas de finales de siglo. En este caso, la antropología estuvo representada por el texto de Bretón, Comas y Contreras (1997).

\section{De los campesinos a la agricultura familiar}

El estudio del campesinado, en cualquiera de sus enfoques, fue perdiendo centralidad en las ciencias sociales a partir de los años 80 . De un lado, porque poco quedaba que ańadir a la importante literatura producida hasta ese momento. De otro, porque los campesinos comenzaban a ser un verdadero vestigio del pasado, arrinconados tras el proceso de modernización de la agricultura iniciado en los años 60 y que culminaba por entonces tras varias décadas de industrialización. La nueva realidad venia marcada por la radical transformación de la agricultura y de la sociedad rural, por el abandono del modelo agrícola autosuficiente y su sustitución por un modelo intensivo, cada vez más dependiente de insumos externos y de la creciente aportación de inputs de capital y tecnología. La modernización completaba una fase que había comenzado a mediados del siglo XX y que suponía la primera de las rupturas sufridas por la sociedad rural, según señala Hervieu (1997).

El nuevo modelo, de carácter desarrollista y productivista, se impuso como dominante en todos los sectores económicos, como necesaria salida para la recuperación de la población tras la II Guerra Mundial. La denominada Revolución Verde, vinculada con una nueva lógica de organización del trabajo, cuyo mejor exponente es el fordismo y la producción masificada, tuvo su expresión en el mundo rural en la introducción de tecnologías para la intensificación de la actividad agraria, la ampliación del área cultivable mediante la creación de regadíos y el inicio del sistema de agroalimentario industrializado. El paradigma de la modernización se convirtió en el más significativo para explicar los cambios fundamentales en el sector agrario, y por extensión en el medio rural, cambios planificados, en muchos casos, desde la misma administración. En España, a imitación de los programa de desarrollo norteamericano, fueron impulsados por la labor de tecnólogos, burócratas, economistas y agrónomos que, en pleno franquismo, crearon el Servicio de Extensión Agraria dentro del Ministerio de Agricultura (Gómez Benito, 1995). 
Por tanto, frente al modelo tradicional, basado en un gran aporte de mano de obra, regido por el ciclo natural de los cultivos y apoyado en una energía manual y natural, comenzó a incentivarse el monocultivo y un agrarismo de mercado centrado en la utilización intensiva del capital y en la especialización productiva (Rubio, 2010). La ciudad se convirtió así en el destino final de la emigración de familias campesinas. Las bases de despoblamiento rural y del envejecimiento de amplías zonas de la España rural quedaban así trazadas, y con ellas la senda de la desagrarización, como el nuevo elemento que definía a la nueva sociedad rural.

Desmontadas las bases sobre las que se habían construido la economía y las formas de vida campesina, poco interés despertaba ya este tema para unos estudios rurales más preocupados por analizar el proceso de su transformación. Los asuntos vinculados al cambio social, el éxodo rural y la emigración se convirtieron en las temáticas centrales de estos estudiosos. Una reorientación de la disciplina que, además, coincidió con un cierto ocaso de los estudios rurales, como si la desvertebración de esta realidad se extendiera también al mismo campo de estudio, que terminó por vincularse con la imagen decadente que lo rural despertaba, frente a la dinámica de los núcleos urbanos.

La ciudad y especialmente las transformaciones operadas en el ámbito urbano, se convertían en nuevos objetos de estudios de los científicos sociales. En este contexto, los estudios campesinos dan paso a los de agricultura familiar, como unidad de análisis válida para explicar los cambios y la evolución en este tipo de unidades económicas (Bretón, 1993). Este concepto explica la coexistencia del modelo agrario modernizado con otros tipos de explotaciones que no pudieron o supieron incorporarse a la nueva tendencia. Refiere a pequeñas explotaciones escasamente competitivas, situadas en la periferia de los mercados y basadas en la mano de obra familiar.

El nuevo concepto es operativo a nivel analítico en la medida que refleja la situación de una categoría de productores agrícolas que habían convertido su actividad en una de las posibles entradas económicas de sus rentas familiares. Son los agricultores a tiempo parcial (De Janvry, 1991), que describe la situación de la mayoría de los pequeños agricultores de los países donde la intensificación agrícola ha tenido una mayor incidencia (Moreno, 2012). La pluriactividad, entendida como la diversificación económica de los miembros del grupo doméstico en distintos sectores laborales, ajenos a la propia explotación se convertiría a partir de entonces en unos de los núcleos centrales de este nuevo enfoque, en la 
medida que profundizaba en la articulación entre la lógica del grupo doméstico y la del mercado.

Se instituye así la dualidad contrastiva entre Peasant y Farmer. El primero, el campesino, vinculado con la forma de agricultura tradicional. El segundo, el agricultor, con una forma capitalizada de agricultura familiar, presente en países de nueva formación. Pequeños propietarios adscritos a explotaciones vinculadas a la nueva oleada de colonización europea desde finales del XIX, por tanto gestionadas desde el principio con una lógica capitalista, pues a pesar de su tamaño reducido y del empleo de fuerza de trabajo doméstica, se integraron rápidamente en las estructuras de mercado, empujados por la lógica del espíritu emprendedor y competitivo de los nuevos colonos (Sacco, 2007; Schneider, 2009).

El concepto de campesino, incapaz de explicar la pervivencia de estos grupos en contextos altamente industrializados, quedaba así restringido a describir un modo de vida vinculado a la sociedad precapitalista. El de agricultura familiar denota más una profesión que un modo de vida. Refiere a una orientación laboral especializada, vinculada con la actividad económica que se realiza y no presenta ninguna otra excepcionalidad con respecto a otros sectores profesionales. Este tránsito hacia la profesionalización del agricultor es otra de las rupturas que Hervieu menciona (1997). Una visión que asemeja al pequeño agricultor al pequeño propietario, profesional de cualquier sector, por tanto inserto en la misma problemática de la sociedad global. No olvidemos este énfasis porque será fundamental para explicar los nuevos cambios de la sociedad rural y con ello, el de los estudios rurales con la entrada del milenio (Bernstein, and Byres, 2001; Berkeley, 2005)5.

A partir de entonces, el enfoque se centra en las distintas estrategias a las que se enfrenta el pequeño-mediano agricultor de nuestros días, frente a su creciente dependencia de los centros de poder agrarios y agroindustriales. Una dependencia determinada por el doble estrangulamiento (squeezed on agriculture, Ploeg 1990), entre los altos costes de los imputs de esta agricultura competitiva y el bajo precio de sus productos en el mercado. Estas estrategias van orientadas a forzar la intensificación de su producción, ya sea por la vía de la diversificación o el de

5 Con todo, reconocidos estudiosos del mundo rural, como es el caso de Ploeg, continúan defendiendo el uso del concepto campesino. $\mathrm{Y}$ es desde esta reificación del viejo concepto como explican su pervivencia, sus estrategias económicas y de resistencia política frente al imperio, representado por los grandes oligopolios agroindustriales (Ploeg, 2010). 
la especialización, lo cual implica, a su vez, una intensificación de su trabajo y el de los miembros de su familia (Bretón, 1993).

En realidad, esta situación describe la posición subalterna que en la actual sociedad tiene todo el sector agrario, cada vez más subordinada a otras esferas de la economía. La agricultura se convierte en un gran mercado de inputs industriales, dependiente de las industrias de productos agrarios (agroindustrias), de incesante expansión a partir de los ańos 80 , y generadoras de grandes oligopolios multinacionales que absorben crecientes proporciones de producción agraria, determinando precios, variedades a producir e incluso procesos y técnicas de producción (Sanz, 1997). Finalmente, otra de las rupturas del mundo rural a la que refiere la ya mencionada obra de Hervieu (1997), quizás una de las más importantes, pues ha determinado la desconexión fundamental entre el campo y el plato, entre agricultura y alimentación (Díaz y Gómez Benito, 2008).

En este contexto de profunda transformación de la sociedad rural tradicional fue cuando la antropología española se olvidó del mundo rural. Terminó así una rica tradición que había sido fundamental en la etapa de formación de la mayoría de los antropólogos de finales de los años 70. De una generación de jóvenes antropólogos que en torno a Juan Vicente Palerm crearon en Cataluńa, el Seminario de los Campesinos (J. Prat, 1992). De un tema que se erigió en el lugar común de las primeras etnografías de aquella generación de antropólogos españoles que realizaron sus tesis doctorales desde mediados de los 70 a finales de los 80, tanto en España, como en América Latina, como atestiguan los casos Contreras en Ecuador (1972), de Giménez en México (1991), de Cucó (1982) en Valencia, o los mismos estudios sobre trabajo y parentesco de Comas y Pujadas (1985) en Aragón, entre otros, por no ser exhaustivos.

A pesar de esta riqueza intelectual y conceptual, la desvinculación entre la temática rural y la antropología fue un hecho en la década de los 90. Sintetizar las causas del ocaso de este campo es ciertamente complejo, pero quizás y simplificando, podríamos decir que la crisis del campesinado se extendió al mismo campo de estudio de la antropología. Desaparecidas muchas de las condiciones económicas sobre las que se construyó este objeto de trabajo, la antropología fue incapaz de trascender hacia otros grupos sociales igualmente rurales, pero que habían permanecido ajenos a nuestra mirada. Y así, como si el campesino hubiera agotado la veta de los estudios rurales por parte de nuestra disciplina, 
muy lentamente el tema se fue eclipsando, para desaparecer casi totalmente con la entrada del nuevo siglo.

Lo rural comenzó a ser percibido como lugar común de tradición e inmovilismo, de modo que su estudio fue considerado poco atractivo para la práctica de una nueva antropología, en la que eclosionaban con fuerza nuevos temas: la antropología urbana, el género, el patrimonio, el turismo, el desarrollo, la alimentación y el gran paradigma que servía de nuevo marco explicativo al cambio y los fenómenos sociales: la globalización.

Lo cierto es que muchos de estos temas de moda seguían teniendo como contexto el escenario rural, que explicaba por ejemplo el valor ascendente de los estudios sobre patrimonio, la alimentación y nuevas formas de consumo, o el papel del género en la nueva agricultura y la economía sumergida, las nuevas segmentaciones del mercado de trabajo y la inmigración. El problema en realidad fue que los antropólogos no supieron ver que esos nuevos temas encontraban también explicación en el profundo proceso de transformación que, lentamente, se estaba fraguando en los territorios rurales desde finales del pasado siglo. Se trataba de un cambio dirigido, al menos por lo que a Europa se refiere, desde la Política Agraria Común (PAC) y sus Programas de Desarrollo Rural. Surgió así el nuevo paradigma para explicar esa nueva ruralidad, una realidad que respondía ahora a nuevos esquemas económicos y sociales, que alejado del inmovilismo con que los estudios anteriores se habían empeñado en analizarla, se mostraba como una realidad dinámica regida por nuevas reglas, nuevos tiempos y nuevos actores (Aguilar, 2012; Pérez Chueca y Aguilar, 2013).

\section{Nuevos escenarios intelectuales para nuevos escenarios rurales}

El error fue seguir confrontando lo rural a lo urbano, mantener esa vieja dicotomía en la que, a esas alturas, ya nadie creía, pero tampoco interesaba rebatir. Por entonces las ciencias sociales en nuestro país estaban tan interesadas en investigar los nuevos fenómenos urbanos, que terminaron por consagrar estos espacios como los propios del cambio, para relegar lo rural a ámbitos ajenos a todo dinamismo (González y Moyano, 2007). Y lo peor es que este estado de

${ }^{6}$ Todavía aparecería en los trabajos ya mencionados de Bretón, Comas y Contreras (1997) y como uno de los temas de estudios de los antropólogos en el libro que en 1996 coordinaron Joan Prat y Ángel Martínez (Aguilar, 1996). 
cosas tuvo su repercusión en la misma reproducción de nuestra disciplina a nivel académico. De ahí la desaparición de la asignatura de Antropología del Campesinado en la mayoría de las licenciaturas y grados de Antropología Social en España.

¿Qué nos estábamos perdiendo volviendo la mirada del mundo rural? Una nueva realidad que nos mostraba unos espacios rurales inscritos en su dimensión local, pero insertos en procesos globales, entendiendo estos últimos en el sentido de nuevas pautas de organización de sociedades avanzadas. Una ruralidad, así, plenamente adscrita a procesos y fuerzas socioeconómicas más amplias, que bien puede ser definida como ruralidad líquida (Camarero y González, 2005), tomando prestado el concepto de modernidad líquida de Bauman (2004). Desde luego esta idea propone un modelo mucho más ajustado a la situación actual de unos escenarios de naturaleza múltiple, donde conviven grupos sociales tradicionales con nuevos empresarios y emprendedores, agentes sociales y una nueva clase de técnicos y políticos que piensan, gestionan y actúan sobre propuestas concretas de desarrollo, a partir de la mediación que les oferta cada uno de los territorios concretos. En definitiva, esta imagen nos sitúa frente a un universo sujeto a itinerarios múltiples y complejos, que lo aleja ostensiblemente de la representación dual a la que nos tenían acostumbrados las clásicas visiones sobre el mundo rural: ruralurbano, dentro-fuera, ciudad-campo, local-global, etc. (González Fernández, 2001).

Elegir esta estrategia teórica supone recurrir al fenómeno largamente estudiado por la literatura científica (Friedman, 2001; Urry, 2003), tal es el hecho de que cualquier aproximación a la realidad social de nuestro tiempo pasa casi de forma inevitable por la referencia ineludible a la globalización, entendida como el contexto que explica los procesos sociales, que difícilmente pueden ser analizados sin su concurso. Desde luego, es obvio que la reestructuración del sistema económico mundial ha tenido su eco en los espacios y sociedades rurales, tendencia que se ha manifestado con especial persistencia desde mediados de los 80 . De esta forma, podemos afirmar que los territorios y la sociedad rural, al igual que el resto de territorios y sociedades, están inmersos en el conjunto de procesos globales que conducen a la constitución de sociedades cada vez más interdependientes e interconectadas, en las que la lógica económica pasa a ser dominante, impregnando todos los ámbitos de la vida social (Bueno y Aguilar, 2003).

Esta nueva realidad ha hecho necesaria la redefinición de los viejos postulados que asociaban ruralidad con actividad agraria y la definían como contrapuesta a lo urbano-moderno-industrializado. Definitivamente, la intensificación y aceleración de los procesos globales se han erigido como los más recientes y sólidos 
argumentos con los que oponerse a las viejas y, ya manidas, dicotomías que enfrentaban lo rural y lo urbano. Podemos afirmar sin paliativos que los cambios globales han terminado por desdibujar las fronteras entre el campo y la ciudad, y si estas fueron siempre poco precisas y bastantes permeables, hoy constituyen un marco de análisis poco adecuado para abordar una realidad social en la que ciudad y campo son sólo piezas dentro de un mismo escenario global (Hoggart y Paniagua, 2001).

A estas alturas parece apropiado afirmar que estamos ante una doble transformación: la del propio objeto de estudio y la de nuestra forma de mirarlo. Pues, en efecto, la emergencia de este nuevo escenario rural, cada vez más alejado de su monocromía agraria, despojado ya de su cobertura de mundo tradicional, ajeno a los cambios y anclado en el pasado, sometido a una lenta transformación emanada desde las ciudades, es ciertamente ya una lectura caduca y desde luego superada por la verdadera realidad de dos espacios, el rural y el urbano, penetrados por una misma lógica económica global.

\section{La ruralidad y sus nuevos significados}

Estas posiciones teóricas para abordar esa nueva ruralidad comenzaron así a desplazar su foco, al estudiar tales transformaciones en su articulación con fenómenos más amplios. Lo rural se convierte así en un enfoque desde el que analizar una realidad mucho más compleja (González y Moyano, 2010). La gran contribución de esta posición será la de vincular el concepto de rural al de construcción social, tal y como ya aparece explícito en la obra colectiva: Constructing the Countryside (Marsden et alii, 1993), en la que se postula la necesidad de enfoques interdisciplinarios para entender los efectos combinados de las distintas fuerzas de cambios a nivel económico, político e institucional.

En esta posición van a coincidir otras propuestas, sustentadas por autores como Lash y Urry (1998), a partir del papel central que en la sociedad postindustrial tiene la Economía de Signos y el Consumo de Significado. Los antecedentes de esta posición lo encontramos en el trabajo de Mormont (1987) que durante los años 80 definía lo rural como parte de las representaciones, tanto espaciales como sociales, de las distintas sociedades en cada momento histórico. Estos nuevos posicionamientos no sólo proporcionaban un nuevo concepto de ruralidad, sino, sobre todo, abría nuevas posibilidades a la hora de estudiar algunos temas claves de los estudios rurales en la actualidad. 
En primer lugar, suponen un avance fundamental a la hora de justificar la existencia de la ruralidad como campo de estudio de tradicional confluencia de sociólogos, antropólogos, geógrafos, agrónomos o economistas. Su importancia radica en el hecho de vincular las representaciones sobre lo rural al contexto de la evolución de las distintas sociedades, lo que supone afirmar que la compresión del espacio rural remite al de la sociedad en su totalidad. Y este énfasis teórico, invertidos los mismos términos del supuesto, nos conduce a otro no menos significativo: profundizar en la concepción de lo rural nos acerca a la comprensión de procesos concretos de las sociedades contemporáneas. En síntesis, mantener este principio, central en las ciencias sociales, vuelve a poner el acento en la funcionalidad epistemológica de los estudios rurales como campo científico especializado. Desde este punto de vista, la investigación sobre los escenarios rurales se convierte en un observatorio privilegiado de la imagen con que la sociedad se representa en cada momento histórico.

En segundo lugar, nos muestra una ruralidad alejada de la tradición —en la que quedan pocos rastros de grupos homogéneos, aislados y autosuficientes-, y nos ofrecen la visión de nuevos paisajes en transformación, con menos especialización agraria y economías muy diversificadas, habitadas por una población crecientemente móvil y conectada con grandes procesos globales (Oliva, 2010).

En tercer lugar, nos permite explicar los nuevos significados de estos territorios, pues la definitiva integración económica de ambos mundos no ha significado la desaparición inmediata del imaginario largamente construido sobre los mismos. Un proceso paradójico, por cuanto se ha ido consolidando al mismo tiempo que las diferencias rural-urbano desaparecían, y por ello, acentuándose la necesidad de producir e incluso de reinventar una imagen de una ruralidad ya inexistente. Será precisamente esta nueva funcionalidad de lo rural, esta redefinición dentro de la sociedad global, la que ha convertido estas áreas en objeto de las nuevas iniciativas económicas, sustentadas desde los nuevos protagonistas del desarrollo rural y configuradas en el centro de las aspiraciones sociales de una clase media, cada vez más receptiva a los mensajes simbólicos asociados a la ruralidad. La paradoja es que las ya caducas dicotomías rural-urbano parecen haber intercambiado sus valores, de modo que estamos ante la aparición de ese idilio rural (Hervieu, 1997), que ha dejado de percibir la ruralidad como problema para convertirla en una necesidad. Y así, frente a su desaparición ahora se busca la preservación, frente a su ocaso, ahora se trabaja por su reinvención (Camarero y González, 2005). 
$\mathrm{Si}$, como decimos, la definición de los espacios rurales ha dejado de construirse sobre la clásica diferenciación con lo urbano, de lo que deberíamos hablar es más de distinción, estrechamente vinculado con los intereses de las nuevas clases medias (Bourdieu,1998). Nos situamos así, frente a un nuevo escenario rural, donde la antigua variable ecológica sigue siendo funcional como criterio espacial, ya que es un hábitat que, en parte, fue construido a partir de su especialización agraria original. Una actividad que, sin ser hoy la fundamental, ha delimitado sus características distintivas, generado unos paisajes específicos y una biodiversidad natural y cultural que conforman en la actualidad el rico patrimonio de estos territorios (Amaya, 2010).

Sin embargo esta diferencia ya no es dual, ni siquiera gradual o continua, es un espacio fluido, líquido (Camarero y González, 2005). Esta diferenciación espacial ha devenido en el de distintividad cualitativa, lo que explica su progresiva especialización en producción de calidad tanto material, como paisajística y de naturaleza, así como de consumo de ocio.

\section{EI desarrollo rural: nuevas reglas para nuevos actores}

El desarrollo rural, ya lo hemos afirmado, constituye el nuevo paradigma para explicar la nueva realidad rural (Ploeg et al., 2000). Hablar de desarrollo rural remite a las recientes políticas que se están ejerciendo en el medio rural europeo desde los últimos cambios de orientación de la PAC. Este proceso iniciado a partir de la década de los 90, vino a cuestionar las medidas modernizadoras impuestas desde los 60 que ya hemos comentado. Los cambios socioeconómicos y culturales de principios de la década de los 90, en un contexto general de prosperidad socioeconómica y de generalización de los comportamientos en el consumo, explican la emergencia de una ruralidad diferente a la tradicional. A nivel global, este cambio refiere a la reestructuración general de la economía, desde una orientación de economías de escala e integración vertical a las regidas por nuevos valores de flexibilización (Piore and Sabel, 1990). Se proyecta así un reacomodo de la PAC acorde con un modelo de consumo alimentario que privilegiaba los productos diferenciales, especialmente aquellos que incluyeran un elevado grado de seguridad.

Las bases de esta nueva era se habían recogidos por vez primera en 1988, en el documento El Futuro del Mundo Rural que, en esencia, conformarían los principios sobre los que se construiría la nueva reforma de la PAC con la que entramos 
en el nuevo milenio, la denominada Agenda 2000. Es en estos momentos cuando se diseñaron las Políticas de Desarrollo Rural, conocidas como el Segundo Pilar de la PAC. El cambio de énfasis fue tan radical que, por primera vez, lo rural y lo agrario dejaron de ser sinónimos y se comenzó a expresar con claridad que el futuro de lo rural no dependía exclusivamente de la actividad agraria, pese a que su participación en la ruralidad seguía siendo esencial (Rubio, 2011).

La ambientalización de la agricultura (Moyano y Paniagua, 1998) fue otro de los ejes de este nuevo modelo de ruralidad. Constituyen el conjunto de medidas tendentes a frenar las externalidades ambientales negativas generadas por la agricultura, fomentar métodos de producción más respetuosos con la naturaleza y dotar al agricultor de una nueva legitimidad social en base a la potenciación de su papel como guardián del medio ambiente.

La actividad agraria, más allá de su función central productiva, se carga de nuevos significados, de valores sustantivos de la nueva sociedad postindustrial, tal y como expresaba el paradigma constructivista más arriba expuesto. El modelo resultante es el de la intensificación capitalista de la agricultura y el diseño de un nuevo mundo rural no centrado exclusivamente en la producción agraria, sino en la diversificación de actividades económicas y en la multifuncionalidad (Gómez-Limón y Barrreiro, 2006).

Se generaliza una visión más amplia del territorio, que deja de ser un mero soporte para el desarrollo de actividades y se convierte en un recurso (Esparcia y Noguera, 1999), capaz de activar todas las dimensiones que contiene, tanto las tangibles-productivas, como las intangibles-no productivas. Se descubren, de golpe, la capacidad de estos espacios para generar externalidades positivas, tanto de tipo ambiental: fomento de la biodiversidad y reducción de contaminación, como en su vertiente territorial, asociadas a la ordenación y administración del territorio, así como en su aportación al mantenimiento de la estructura demográfica y de los mercados de trabajo (Rubio, 2011).

Esta ruralidad se proyecta más allá de la actividad meramente agraria para descubrir las distintas sinergias que este sector genera sobre el territorio, de tipo paisajístico, ambiental, patrimonial o demográfico. La actividad agrícola, desde esta nueva perspectiva desempeńa un valor económico fundamental que, más allá de ser mera productora de alimentos, genera además actividades en distintos sectores: agroindustria, abastecimiento agrario, $\mathrm{u}$ otras pequeñas industrias derivadas: textiles, cueros, queserías, cosméticas, farmacéutica, etc. 
Resulta ilustrativo recordar que el mundo rural siempre fue multifuncional, en la medida que el mismo ciclo biológico de la agricultura tradicional regulaba la inserción laboral intermitente de sus miembros a otros sectores productivos. Este modelo empujaba además a la autosuficiencia del grupo para proveerse de bienes y de servicios complementarios derivados de la misma explotación agroganadera, de modo que la confección de textiles, pieles, quesos y otros productos especializados era una necesidad consustancial a este sistema tradicional. Sólo que esa pluriactividad refería a necesidades internas de organización del grupo doméstico, en tanto que el término de multifuncionalidad responde ahora a la generación de diversos sectores económicos a nivel del territorio.

\section{Competitividad territorial, innovación, redes y nuevos nichos de mercado}

El enfoque territorial se ha convertido en el principio definitorio de la nueva sociedad rural, por cuanto constituye el eje central de la Política de Desarrollo Rural. Contaba con el antecedente de las Iniciativas Comunitarias Leader ${ }^{7}$ (Ray, 2002) que, desde 1991 constituyó el modelo a seguir para los programas orientados a la dinamización del mundo rural. Su filosofía, basada en la diversificación de actividades económicas, y su enfoque y sistema de gestión bottom-up lo han instituido por derecho propio en una de las acciones fundamentales de las Políticas de Desarrollo, cuyo papel protagonista seguirá siendo fundamental en la nueva reforma de la PAC que se aplicará en el periodo 2014-2020 (Esparcia y Escribano, 2012; Massot, 2012).

No olvidemos que estamos ante un fenómeno de transformación de los espacios rurales que está perfectamente diseñado desde instancias supranacionales, concebido como la única estrategia posible, al día de hoy, para que estos espacios puedan afrontar los retos económicos asociados a la globalización. Pero es evidente que estos posicionamientos han requerido de la creación de una novedosa armazón institucional, que ha rediseñado el nuevo mapa de la ruralidad, ubicando a nuevos actores y agencias en el mapa político de toma de decisiones, correspondiente a la nueva división administrativa en la que se ha replanteado el escenario rural. Rural».

${ }^{7}$ Leader son las siglas en francés de «Liaisons entre activités de Developement de L'Economie 
A esa realidad responde la aparición de nuevas agencias de desarrollo cuyo papel ha sido el de gestionar y planificar las distintas acciones del desarrollo a nivel de territorio. Conforman así una nueva gobernanza que se corresponde con nuevas funciones productivas, turísticas, ecológicas, patrimoniales de estos espacios. Estas nuevas divisiones administrativas comarcales, basadas en antiguas demarcaciones culturales e históricas o recreando nuevas, y gobernadas por los Grupos de Acción Local (GAL) se han erigido en los nuevos vehículos institucionales del desarrollo rural europeo. Su papel ha sido crucial, por cuanto han liderado cuantas iniciativas se han proyectado en estos espacios, actuando como agencias de intermediación, tanto política como financiera, entre los Programa de Desarrollo Rural, los Fondos de financiación europeos, las distintas administraciones estatales y regionales y el nivel local, así como de interlocutores con los ciudadanos (Pérez y Aguilar, 2013). De este modo, los técnicos de desarrollo rural, conforman uno de los grupos representativos de los nuevos actores de estos escenarios rurales.

En estos momentos, y tras más de 20 años de implementación de políticas de desarrollo, podemos decir que la transformación de estos escenarios es un hecho. Esta realidad ha consagrado tanto el enfoque Leader como el territorial como partes sustantivas e irrenunciables de estas acciones. Si bien es verdad que estamos en un momento crucial: el diseño de una nueva PAC que verá la luz en 2014 y que, con horizonte 2020, pretende reformular y sobre todo corregir, alguna de las debilidades de la anterior. En esencia, una vuelta al valor central de la actividad agraria, como sector prioritario del mundo rural. De este modo, el enfoque territorial se desliza ahora hacia el que podemos denominar como agro-territorial. Ello supone el planteamiento de un desarrollo policéntrico, que implica una mayor integración rural-urbana, y un deslinde de las Políticas de Desarrollo, a nivel de financiación de la PAC, que ahora se concentra en las ayudas al sector agrario, integrando las agendas de los Programas de Desarrollo en iniciativas concretas de carácter territorial, ya sea urbanas o rurales. Se trata de reconocer definitivamente que no existe una dicotomía entre los espacios urbanos y rurales (Moyano et al., 2011) y de fomentar un enfoque estratégico para la cohesión territorial y el mantenimiento de la vitalidad de las áreas rurales ${ }^{8}$.

Los elementos en los que se sustentan estas nuevas directrices son, en esencia, el mantenimiento y/o reforzamiento de los elementos sociales, ambientales

${ }^{8}$ Sus principios están recogidos en los documentos elaborado durante los últimos años: la Estrategia Territorial Europea, el Libro Verde de la Cohesión Territorial, o la Agenda Territorial Europea. 
y territoriales hasta ahora reseñados. Las preocupaciones ambientales destacan sobre otras, en la medida que responde a nuevas preocupaciones ya plenamente asumidas tanto por las agendas política como por los consumidores, por cuanto están vinculadas a la seguridad alimentaria. De ahí el incremento de las ayuda agroambientales, y el apoyo expreso a la agricultura ecológica (Lozano, 2011; Lozano, Aguilar, 2011), así como el apoyo a los circuitos cortos de comercialización (González Calo et al., 2012; Sevilla Guzmán et al., 2012).

A partir de ahí, y como no podría ser de otro modo, se insiste en los modelos ya ensayados con éxito en estos espacios rurales, tanto en la relativo a la generación de redes económicas y sociales competitivas a distintos niveles: locales, regionales e internacionales (Marquardt et al., 2012;), como en términos de apoyo a la innovación y a las nuevas tecnologías, como sustrato vinculados a las nuevas necesidades de una nueva economía (Esparcia y Escribano, 2012; Pérez y López, 2014).

\section{Natural, calidad y rural}

Este contexto explica la creciente especialización de la ruralidad en productos de calidad, cuyo valor añadido reside precisamente en su vinculación con un territorio concreto. Los elementos consustanciales de su ecología, su tradición, $s u$ historia se incorporan, como elementos distintivos, a esos productos locales (Lozano y Aguilar, 2010), y es a partir de esa transferencia de significados sociales, como logran su características diferenciales de en los mercados globales.

A esta tendencia obedece la actual proliferación de los marcas de calidad que vienen a avalar las cualidades diferenciales o la renta de especificidad que determinados alimentos poseen (Tregear et al., 2007). En este proceso destacan figuras como las Denominación de Origen Protegida (DOP) e Indicación Geográfica Protegida (IGP), o la de Agricultura Ecológica (AE), como marcas con un nivel alto de certificación y con rango de reconocimiento europeo, amparadas por unas regulaciones muy estrictas, lo que garantiza la vinculación del producto con un ecosistema específico, con unas materias primas singulares, con un saber-hacer y/o unas técnicas de elaboración concretas. (Amaya y Aguilar, 2012; Villafuerte et al., 2012).

La búsqueda de una posición favorable en los mercados es hoy una de las constantes de esta nueva economía rural. A este tipo de acciones van dirigidas 
precisamente muchos de los programas de desarrollo rural, que han tendido a crear marcas territoriales, más allá de las agroalimentarias. Este reencuentro ha propiciado que, de forma paulatina, se esté fomentando una mayor vinculación entre alimento y producto local, entre productor y consumidor. La naturaleza, el territorio, la historia y la cultura constituyen así en los vínculos que conectan con la calidad del producto, en los garantes de su valor añadido de cara al mercado.

Es cierto que este fenómeno ha generado cierta esquizofrenia por labelizar el mundo rural, lo que nos habla de los peligros de esa progresiva tendencia a musealizar la realidad (Bowen, 2011). Porque más allá de los productos agroalimentarios, los mismos territorios rurales se constituyen actualmente como marcas, bien ligadas a las especificidades ecológicas del territorio (Marca Parque Natural), o a las técnicas tradicionales de elaboración de un determinado producto local (Marca Artesana), o a las excelencias turísticas de las zona (Marca Q de calidad) (López y Aguilar, 2013).

Pero también es importante recordar que en esa vuelta hacia prácticas, manejos, técnicas y saber-hacer tradicionales — ahora readaptados a las nuevas exigencias de gusto, estéticas y controles de salubridad—, residen muchas de las innovaciones de estos productos (Amaya y Aguilar, 2013). Unas innovaciones que se sustentan en la precisa recombinación de recursos, coordinación y (re) modelación de las bases materiales y sociales y el renovado uso del capital ecológico, social y cultural de estos territorios rurales (Ploeg et al., 2000). El encuentro entre la tradición y la innovación es el elemento sustentador de la excelencia y la calidad estos nuevos productos locales, y por extensión de estos nuevos escenarios rurales.

\section{Conclusiones}

En este texto hemos presentado las principales transformaciones de la ruralidad, profundizando en algunas de las claves que la definen en la actualidad, para vincularla a cambios operados a nivel global. Este recorrido nos muestra un mundo rural en permanente cambio, que ha ido modificado la misma perspectiva de análisis de los estudios rurales hasta la actualidad.

Hemos señalado que la industrialización agraria y la modernización, acaecidas desde finales del XIX hasta mediados del pasado, marcaron las principales rupturas de una ruralidad que vio desaparecer las formas de vida campesina, para 
dar paso a un modelo de agricultura intensiva para el mercado. El fracaso de este paradigma determinaría el nuevo modelo rural, caracterizado por el tránsito de una agricultura intensiva y estandarizada, a un sector agrario multifuncional centrado en la búsqueda de la distinción y la calidad alimentaria. En este contexto, las directrices de la PAC y las Políticas de Desarrollo Rural han sido decisivas para consagrar una nueva realidad construida sobre nuevos valores de referencia.

Asimismo, hemos descrito el proceso de resignificación de lo rural y su papel dentro de la nueva sociedad postindustrial, considerándolo como la clave definitoria de esta realidad. Sus funciones ambientales, territoriales, paisajísticas y patrimoniales, conviven hoy con su primigenio papel como proveedor de alimentos, sin olvidar el peso de las nuevas dimensiones de desarrollo territorial como son el capital social, el tejido institucional, las infraestructuras y servicios de telecomunicaciones, y el tejido empresarial.

Podemos terminar afirmando que esta nueva ruralidad europea es una recombinación de diversas rupturas y reencuentros, producto de un proceso muy complejo que se ha ido sucediendo a lo largo de la segunda parte del siglo XX. El resultado actual es una realidad enteramente nueva, que contiene ciertas dosis de tradición releída con nuevos códigos de innovación, construida por nuevos actores y que responde a los nuevos tiempos de la sociedad postindustrial.

\section{Bibliografía}

Aguilar, E. (1996). «Campesinos», en Joan Prat y Ángel Martínez (eds.), Temas de Antropología Cultural. Barcelona: Ariel, pp. 113-127.

- (2005). «Productos locales, mercados globales: nuevas dinámicas en el medio rural, en M. García Docampo (ed.). Perspectivas teóricas en desarrollo local. A Coruña: Netbiblo, pp. 405-423.

- (2012). «Nuevos tiempos, nueva reglas, nuevos actores: de campesinos a rurales». En J. Contreras, J. J Pujadas y J. Roca (eds.) Pels camins de l'etnografia. Un homenatge a Joan Prat. Tarragona: Ed. Rovira y Virgili. pp. 129-140.

Aguilar, E.; Feixa, C. y Melis, A. (2000). «Tradiciones y escenarios actuales de la Antropología en España». Nueva Antropología, vol. XVII, 58, pp. 101-122.

AмaYa, S. (2010). «Patrimonio cultural y desarrollo rural como respuesta local ante procesos globales.», en J. Marcos, S. Rodríguez y E. Luque (eds.), Nos-Otros: Miradas antropológicas sobre la diversidad. Badajoz: Asamblea de Extremadura. pp. 889-902. 
Amaya, S. y Aguilar, E. (2012). «La construcción de la calidad alimentaria: tradición, innovación y poder en las DOP del jamón ibérico en España», en Revista de Economia Agrícola (REA). Sao Paulo (Brasil), vol. 59, n. 2, pp. 39-52.

Appadurai, A. (ed.). (1986). La vida social de las cosas. Perspectivas culturales de las mercancías. México: Grijalbo.

Bauman, Z. (2004). Modernidad líquida. México: F. C. E.

Bеск, U. (1998). ¿Qué es globalización? Falacias del globalismo, respuestas a la Globalización. Madrid: Paidos.

Berkeley, H. (2005). New Rural Economy: Change, Dynamism and Government Policy. London: Institute of Economic Affairs.

Bernstein, H. and Byres, T. (2001). «From Peasant Studies to Agrarian Change». Journal of Agrarian Change, vol. 1, no , pp. 1-58.

Bowen, S. y De Master, K. K. (2011). «New rural livelihoods or museums of production? Quality food initiatives in practice». Journal of Rural Studies, vol. 27, pp. 73-82.

Bourdieu, P. (1998). La distinción. Criterio y bases sociales del gusto. Madrid: Taurus.

Brandes, S. (1991). «España como 'objeto' de estudio: reflexiones sobre el destino del antropólogo norteamericano en España», en M. Cátedra (ed.), Los españoles vistos por los antropólogos. Madrid: Júcar, pp. 231-249.

Bretón, V. (1993). «¿De campesino a agricultor? La pequeña producción familiar en el marco del desarrollo capitalista». Noticiario de Historia Agraria, no 5, pp. 127-159.

Bretón, V.; Comas, D. y Contreras, J. (1997). "Cambio social en la agricultura familiar española», en Gómez Benito, Cristóbal y González, Juan Jesús (eds.), Agricultura y Sociedad en el cambio de Siglo. Madrid: Mc Graw-Hill, pp. 652-672.

Bueno, C. y Aguilar, E. (Coords.). (2003). Las expresiones locales de la globalización. México y España. México: CIESAS, Universidad Iberoamericana y Ed. Porrúa.

Caldentey, P. y Gómez Muñoz, A. C. (1996). «Productos típicos, territorio y competitividad». Agricultura y Sociedad, no 80-81, pp. 57-82.

Camarero, L. y González, M. (2005). «Los procesos recientes de transformación de las áreas rurales españolas: una lectura desde la reestructuración ampliada. Sociología. Revista de la Faultade de Letras. Universidade de Porto, vol. 15, pp. 95-123.

Chayanov, A. (1974). [1925]. La organización de la unidad doméstica campesina. Buenos Aires. Nueva Visión.

Cloke, P.; Marsden, T. and Mooney, P. (2006). The Handbook of Rural Studies. London: Sage. 
Comas, D. y Pujadas, J. J. (1985). Aladradas y güellas. Trabajo, sociedad y cultura en el Pirineo Aragonés. Barcelona: Anthropos.

Contreras, J. (1972). Adivinación, ansiedad y cambio social en Chinchero (Perú). Barcelona: Secretariado de Publicaciones de la Universidad de Barcelona.

Cucó, J. (1982). La tierra como motivo. Propietarios y jornaleros en dos pueblos valencianos. Valencia: Edicions Alfons el Magnànim.

Diaz, C. y Gómez Benito, C. (2008). Alimentación, consumo y salud. Barcelona: Obra Social Fundación «La Caixa».

Durkheim, E. (1995). (1893). La división social del trabajo. Madrid: Ed. Akal.

Esparcia, J. y Noguera, J. (1999). «Reflexiones en torno al territorio y al desarrollo rural», en Ramos Real, (ed.), El Desarrollo Rural en la Agenda 2000. Madrid: MAPA, pp. 9-44.

Esparcia, J. y Escribano, J. (2012). «La dimensión territorial en la programación». Anales de Geografia, vol. 32, no 2, pp. 227-252.

Espeitx, E. (1996). «Los nuevos consumidores o las nuevas relaciones entre campo y ciudad a través de los productos de la tierra». Agricultura y Sociedad, no 80-81, pp. 83-116.

Foster, G. M. (1951). «Report on an Ethnological Reconnaissance of Spain». American Anthropologist, vol. 53, no 3, pp. 311-325.

Friedman, J. (2001). «Sistema global, globalización y parámetros de la modernidad», en Friedman (ed.), Identidad cultural y proceso global. Buenos Aires: Amorrortu, pp. 297-352.

Galeski, B. (1977). Sociología del campesinado. Barcelona: Península.

Garrido, F. y Moyano, E. (2004). «Agricultura, sociedad y medio ambiente en la España actual», en M.a J. Marrón y G. García (Coords.), Agricultura, Medio Ambiente y Sociedad. MAPA. Madrid, pp. 29-56.

GimÉNez, C. (1991). Valdelaguna y Coatepec: permanencia y funcionalidad del régimen comunal agrario en España y México. Madrid: MAPA.

Gómez Benito, C. (1995). Políticos, burócratas y expertos. Un estudio de la política agraria y la sociologia rural en España. Madrid: Siglo XXI.

Gómez Benito, C. y GonzÁlez, J. J. (eds.). (1997). Agricultura y Sociedad en la España contemporánea. Madrid: CIS.

- (2002). Agricultura y Sociedad en el cambio de Siglo. Madrid: Mc Graw-Hill.

Gómez-Limón, J. A. y Barreiro, J. (Coords.) (2006). La multifuncionalidad de la agricultura en España. Madrid: MAPA. 
Gómez Mendoza, J. (2001). «Las nuevas funciones socioeconómicas y medioambientales de los espacios rurales», en García Pascual, F. (Coord.) El mundo rural en la era de la globalización: incertidumbres y potencialidades. Madrid: MAPA.

González, M. y Moyano, E. (2007). «Sociología rural», en Manuel Pérez Yruela (Comp.) La Sociología en España. Madrid: CIS, pp. 107-141.

González Calo, I.; De Haro, T.; Ramos, E. y Renting, H. (2012). «Circuitos cortos de comercialización en Andalucía: un análisis exploratorio». Revista Española de Estudios Agrosociales y Pesqueros. no 232, pp. 193-230.

GonzÁlez Fernández, M. (2001). Sociología y ruralidades: la construcción social del desarrollo rural en el valle de Liébana. Madrid: MAPA.

Hervieu, B. (1997). Los campos del futuro. Madrid: MAPA.

Hobsbawm, E. J. y Alavi, H. (1976). Los campesinos y la politica, y las clases campesinas $y$ las lealtades primordiales. Barcelona: Anagrama.

Hoggart, K. y Paniagua, A. (2001). "What rural restructuring?». Journal of Rural Studies, vol. 17, pp. 41-62.

Inglehart, R. (1971). «The Silent Revolution in Post-Industrial Societies». American Political Science Review, no 65, pp. 991-1017.

JANVry et al. (1991). "Peasant Household Behaviour with Missing Markets: Some paradox Explained». The Economic Jorunal, no 1, p. 1.400-1.417.

Kaustsky, H. (1974). (1899). La cuestión agraria. Estudio de las tendencias de la agricultura moderna y de la política agraria de la socialdemocracia. Barcelona: Laila.

Lash, S. and Urry, J. (1998). Economías de Signos y Espacios. Buenos Aires: Amorrutu.

Lenin, V. I. (1975). (1899). El desarrollo del capitalismo en Rusia. Madrid: Tecnos.

López, I.; Aguilar, E. (2013). «La nueva economía rural europea. Especialización territorial de calidad en la Isla de Texel y la Sierra de Cádiz». Gaceta de Antropología, no 29 (2), artículo 04.

LozAno, C. (2011). El sabor de la naturaleza: agricultura ecológica en Parques Naturales andaluces. Sevilla: Fundación Blas Infante.

Lozano, C., y Aguilar, E. (2010). «Natural, tradicional y de la tierra. La promoción de la calidad agroalimentaria en los nuevos espacios rurales andaluces», en Soler, M. y Guerrero, C. (Coords.), Patrimonio cultural en la nueva ruralidad andaluza. Sevilla: IAPH, pp. 126-139.

- (2012). «Territorialising organic production: collective actions and public policies in Andalusia». Sviluppo Locale, vol. XV, no 37-38, pp. 45-66.

Magdoff, F.; Foster, J. y Buttel, F. (Ed). (2000). Hungry for Profits: the Agribusiness Threat to Farmers, Food, and the Environment. New York: Monthly Review Press. 
Marchenay, P. et Berard, L. (2004). Les produits de terroir, entre cultures et règlements. Paris: CNRS.

Marquardt, D.; Möllers, J. and Buchenrieder, G. (2012). «Social Networks and Rural Development: LEADER in Romania. Sociologia Ruralis, vol. 52, $\mathrm{n}^{\circ}$, pp. 398-431.

Marsden, T.; Murdoch, J.; Lowen, P.; Munton, R., and Flynn, A. (1993). Constructing the Countryside. London: UCL Press.

Martins, M. and Marques, M. (2006). «Is agricultural policy promoting a new role for farmers?». Journal of Policy Modelling, vol. 28, no 8, pp. 847-860.

Marx, K. (1975). [1867]. El Capital. Madrid: Siglo XXI.

Massot, A. (2012). «Los mecanismos de la PAC 2020, principales vectores del proceso de reforma en curso». Revista española de estudios agrosociales y pesqueros, $\mathrm{n}^{\circ} 232$, pp. 13-68.

Mauleón, J. R. (2010). «Mercados de agricultores en Espańa. Diagnóstico y propuestas de actuación». CEDDAR, no 23, pp. 1-19.

Mintz, S. W. (1973). «A note on the Definition of Peasantries». Journal of Peasant Studies. I (1): 91-106.

Moreno, O. (2012). «Revisando las categorías de análisis de la agricultura familiar un caso de estudio del Campo de Cartagena». Revista española de estudios agrosociales y pesqueros, no 232, pp. 101-130.

Mormont, M. (1987). «Rural nature and urban natures». Sociologia Ruralis, vol. 27, $\mathrm{n}^{\circ} 1$, pp. 1-20.

Moyano, E. et al. (2011). Foro de Cohesión Territorial. Córdoba: IESA.

Moyano, E. y Paniagua, A. (1998). «Agricultura, espacios rurales y medio ambiente». Revista Internacional de Sociologia (RIS), vol. 19-20, pp. 127-152.

Oliva, J. (2010). "Rural Melting-pots, Mobilities and Fragilities: Reflections on the Spanish Case». Sociologia Ruralis, vol. 50, no 3, pp. 277-295.

Palerm, A. (1976). Modos de Producción y Formaciones Socioeconómicas. México: Ed. Gernika.

- (1987). «Antropólogos y campesinos. Los límites del capitalismo», en Angel Palerm, Antropología y Marxismo. México: CIESAS, pp. 161-185.

Pérez, A. y Aguilar, E. (2013). «Aportaciones al análisis comparativo entre modelos de desarrollo rural en Europa. Gazeta de Antropología, 29 (2).

PÉrez, A. y López, I. (2014). «Ahora somos del consorcio». La construcción de nuevas comarcas de desarrollo rural en Andalucía: desarrollo rural y transformación social», en Inguruak, Revista Vasca de Sociología y Ciencia Política, pp. 2077-2092). 
Piore, M. J. y SABel, C. (1990). La segunda ruptura industrial. Madrid: Alianza.

Pitt Rivers, J. (1954). The People of the Sierra. London: Weidenfeld and Nicholson.

Ploeg, J. D. et al. (2000). «Rural Development: From Practices and Policies towards Theory». Sociologia Ruralis, vol. 40, no 4, pp. 391-407.

Ploeg, J. D. (2003). The virtual Farmer. The Virtual Farmer: Past, Present and Future of the Dutch Peasantry. A. A. Assen: Van Gorcum Ltd.

- (2010). Nuevos campesinos: campesinos e imperios alimentarios. Barcelona: Icaria.

Prat, J. (1992). Antropología y Etnología. Madrid: Ed. Complutense.

RaY, C. (2000). «The EU Leader Programme: Rural Development Laboratory». Sociologia Ruralis, vol. 40, no 2, pp. 163-171.

Redfield, R. (1947). "The folk Society». The American Journal of Sociology. Vol. LII, no 4, 293-308.

Rubio, P. (2010). «Modelización de los cambios y evolución reciente del sistema rural español». $A G E R, \mathrm{n}^{\circ} 54$, pp. 203-235.

SACCO, F. (2007). "Pluriactividad y agricultura familiar en Brasil: el caso de Río Grande do Sul». Revista de la CEPAL, no 93, pp. 157-173.

Sahlins, M. (1972). Las sociedades tribales. Barcelona: Nueva Colección Labor.

SAnz, J. (1997). «El sistema Agroalimentario español. Cambio, estructural, poder de decisión y organización de la cadena alimentaria». En Gómez Benito, Cristóbal y González, Juan Jesús (Coords.) Agricultura y sociedad en la España contemporánea. Madrid: CIS, pp. 355-397.

Schneider, S. (2009). "La pluriactividad en el medio rural brasileño: características y perspectivas para la investigación, en Grammont H. C. y Martínez L. (Comp.). La pluriactividad en el campo latinoamericano. Cuenca: FLACSO, Ecuador, pp. 208-237.

Sevilla Guzmán, E. (1979). La evolución del campesinado en España. Barcelona: Península.

- (1984). «A modo de presentación: Anotaciones para el pensamiento social agrario en España», en Sevilla Guzmán (Coord.), Sobre agricultores y campesinos. Estudios de Sociología Rural de España. Madrid: Servicio de Publicaciones Agrarias, pp. 9-19.

Sevilla Guzmán, E.; Soler, M.; Gallar, D.; Vara, I.; Calle, A. (2012). Canales cortos de comercialización alimentaria en Andalucía. Sevilla: Fundación Centro de Estudios Andaluces.

Shanin, T. (1983). La clase incómoda. Sociología politica del campesinado en una sociedad en desarrollo. Madrid: Alianza. 
Tregear, A.; Arfini, F.; Belletti, G. y Marescotti, A. (2007). «Regional foods and rural development: The role of product qualification». Journal of Rural Studies, vol. 23, pp. 12-22.

Urry, J. (2003). Global Complexity. Cambridge: Polity.

Villafuerte, A.; Gómez, A. C. y De Haro, T. (2012). «El concepto del «valor marca» aplicado a los signos de calidad con indicación geográfica». Revista española de estudios agrosociales y pesqueros, $\mathrm{n}^{\circ} 232$, pp. 131-164.

Wolf, E. R. (1971). Los campesinos. Barcelona: Nueva Colección Labor.

Recibido: 20/12/12

Aceptado: 25/03/13 